\title{
The Iron Project: \\ Atomic Data for Fe I-Fe VI
}

\author{
MANUEL A. BAUTISTA, SULTANA N. NAHAR, \\ JIANFANG PENG, ANIL K. PRADHAN, AND HONG LIN ZHANG
}

Department of Astronomy, Ohio State University, 174 West 18th Avenue, Columbus, OH 43210, USA

Recent progress in large scale computations of photoionization cross sections, radiative transitions probabilities, and collision strengths for the lowest ionization stages of Iron, i.e., Fe I-VI, are reported. These results are part of an international collaboration called the IRON Project. The present results exhibit large differences with respect to data currently in use and is expected to be of importance in the study of astrophysical objects in the UV and the EUV.

\section{Introduction}

One of the major limitations in the analysis of observations of astronomical objects is the availability of reliable atomic data. Accurate photoionization cross section are necessary in the calculation of the photoionization and emissivity models, the opacities, and recombination rates for the ionization balance of the plasmas considered. Electron excitation cross sections and transition probabilities are required for the interpretation of emission spectra.

The IRON Project (IP) is an international collaboration devoted to large scale computations of electron-impact excitation cross sections, as well as photoionization cross sections and radiative transition probabilities for Iron peak elements and ions (Hummer et al. 1993). These data will complement the extensive radiative computations by the Opacity Project (OP; Seaton et al. 1992) which is currently available with the data server TOPbase (Cunto et al. 1993).

Despite the considerable interest in the low ionization stages of Iron in laboratory and astrophysical plasmas, accurate atomic data for these species was not available. This is because the calculations for these ions are particularly difficult, mainly owing to the complex electron-electron correlation effects involved. Another difficulty is the large number of atomic states, the coupling effects, and the complex resonance structures that need to be considered for an accurate treatment. For example, accurate representation of autoionizing resonances and bound-bound transition in $\mathrm{Fe} I \mathrm{~V}$ and $\mathrm{Fe} \mathrm{V}$ are necessary in the computation of monochromatic opacities in the EUV spectra of hot, young white dwarfs (Vennes 1995; Pradhan 1995 in this issue).

In the present paper, we report on the latest advances in the IP for the lowest ionization stages of Iron, Fe I-VI, whose lines are very prominent in the UV and EUV spectra of many objects.

\section{Radiative Data for Fe I-Fe V}

Extensive calculations of photoionization cross sections and transition probabilities for Fe I-VI are being carried out by the Ohio State group. For these calculations, multiconfiguration coupled-channel eigenfunction expansions for the electron+ion system are considered using the R-matrix method as developed for the OP and the IP.

Previous calculations for these ions were carried out in the central field type approximation by Reilman \& Manson (1979) and Verner et al. (1993); however, these computations 


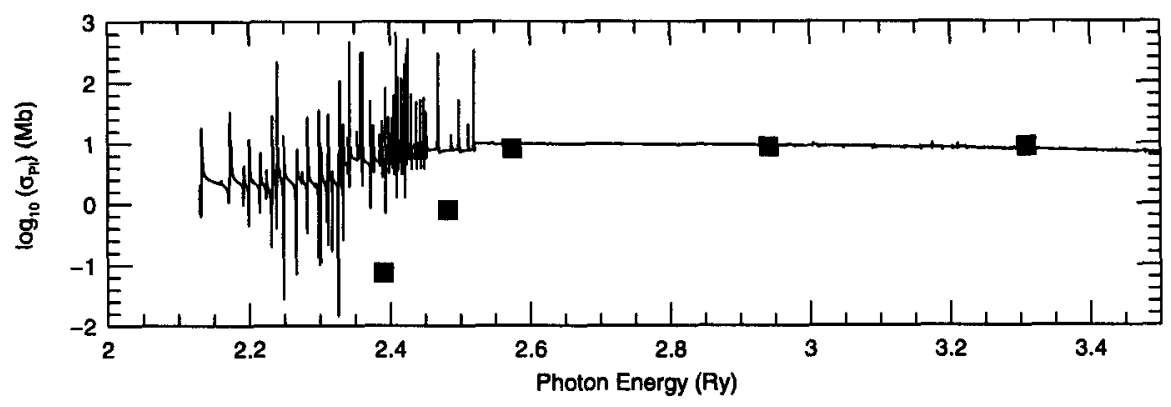

Figure 1. Photoionization cross section of the ground state $3 d^{6}\left({ }^{5} D\right)$ of Fe III: solid curve = present; filled dots = Reilman \& Manson (1979).

do not take account for the coupling effects and resonances that are of considerable importance for atomic systems such as the low ionization stages of Iron (e.g. Nahar \& Pradhan 1994). Earlier R-matrix calculations for Fe I-VI were also done by Sawey \& Berrington (1992) using much smaller close-coupling expansions than what is considered in the present work; this results in important configuration interaction missing from their calculations (for example, their calculations do not obtain the ground state of $\mathrm{Fe} \mathrm{I}$, and in general their photoionization cross sections are up to an order of magnitude, or more, lower).

We have recently calculated the photoionization cross sections for the ground state of neutral Iron (Bautista \& Pradhan 1995a). A comparison of our results with those reported by Reilman \& Manson (1979) and Verner et al. (1993) is shown by Pradhan (1995 this issue). It is found that the previous computations for Fe I do not obtain the dominant contributions to the cross section, owing to the absence of coupling effects, which results in a difference with respect to our results of over three orders of magnitude for photon energies of up to about 1 Rydberg. Photoionization cross sections of Fe II have been reported by Nahar \& Pradhan (1994), where differences of up to an order of magnitude are found in the ground state cross section with respect to the results by Reilman \& Manson (1979) and Sawey \& Berrington (1992).

Radiative transition probabilities ( $f$-values) have been calculated for Fe II for 35,941 LS multiplet transitions. For the transitions for which experimental values are available, the calculated f-values agree generally to within 10\% (Nahar and Pradhan 1994). Recently, radiative transition probabilities for 21,589 dipole fine structure transitions, and over 19,000 transitions between LS multiplets, in Fe II have been reported by Nahar (1995) using experimentally observed energies and theoretical line strengths. For all transitions compared, the present lifetimes and f-values compare better with the experimentally observed values than the Kurucz $(1981,1991)$ data for Fe II. Transition probabilities are being calculated for Fe I, Fe III, Fe IV and Fe V.

Photoionization cross sections of Fe III-V are currently in progress. However, ground state cross sections of Fe III and Fe V have been obtained and they exhibit considerable differences with respect to earlier computations. In Fig. 1 we show the ground state photoionization cross section of Fe III, $3 d^{6}{ }^{5} D$, together with the results by Reilman \& Manson (1979). It is noticed that their results neglect the important contribution of the resonances near the threshold and underestimate the cross section at low photon energies by several factors; however, the agreement between the background cross sections in the higher energy regions is very good. 

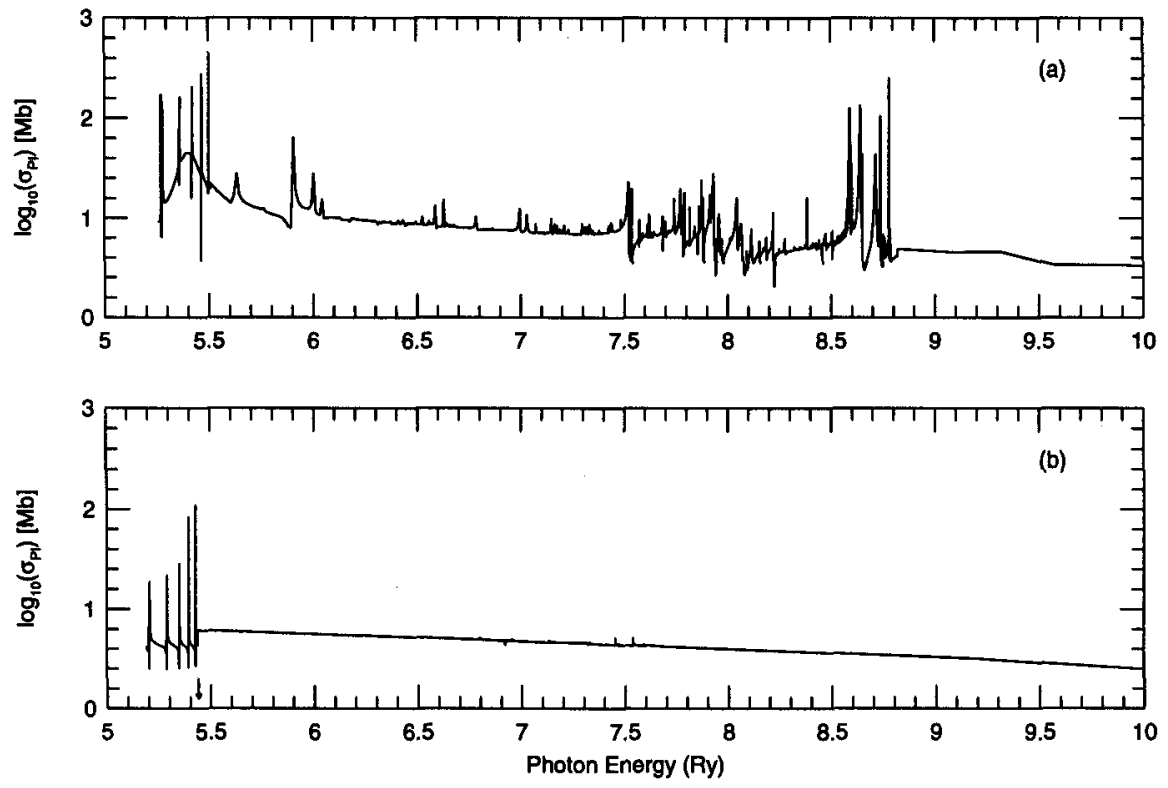

FIGURE 2. Photoionization cross section of the ground state $3 d^{4}\left({ }^{5} D\right)$ of $\mathrm{Fe}$ V: (a) present; (b) Sawey \& Berrington (1992).

The ground state photoionization cross section of $\mathrm{Fe} \mathrm{V}$ is presented in Fig. 2(a), while the earlier results by Sawey \& Berrington (1992) are shown in Fig. 2(b). The large number of additional terms in our close-coupling expansion gives rise to more extensive resonance structures and enhance the background cross section by over a factor of two.

The IP work at Ohio State is now being extended to also calculate not only the total but also the partial photoionization cross sections into excited states of the residual ion. These data are required for non-LTE spectral models and the calculation of total and partial electron+ion recombination rate coefficients (e.g. Nahar and Pradhan 1995).

\section{Collision Strengths for Fe II-Fe VI}

The main emphasis of the IP is on the computation of collision strengths for astronomically important atoms and ions. Among these, the low ionization stages of Iron are of particular importance since their rich atomic structures give rise to a large number of transitions that are seen in many objects, and in almost all the regions of the spectrum. The dipole allowed transitions in these systems in particular correspond to a multitude of observed UV and EUV lines, $\lambda \lambda 3000-200 \AA$. Accurate collisional data for these ions must include fine structure, and therefore needs to take into account relativistic effects, as well as the extensive electron-electron correlation effects.

Considerable effort has been dedicated to the calculation of collisional data for Fe II, and a data set of Collision strengths and rate coefficients for 142 levels and 10,011 associated fine structure transitions, including many transitions observed in the UV, is currently available (Pradhan \& Zhang 1993; Zhang \& Pradhan 1995a). These data are expected to be more accurate than earlier results by Nussbaumer \& Storey (1980) and 
Keenan et al. (1988). Other calculations for some additional transitions (and which provide a check on the accuracy of the present data set) are being carried out (Bautista \& Pradhan 1995b).

Collisional data for Fe III have recently been obtained for 219 even parity levels and 23,871 associated fine structure transitions. These data exhibit differences of up to a factor two with respect to earlier calculations by Berrington et al. (1991) for some of the low lying forbidden transitions. A detailed study of relativistic and electron correlation effects has been carried out in these calculations which suggests that the present data should be accurate to about $10-30 \%$ (Zhang \& Pradhan 1995b). A more extensive calculation for this ion which include odd parity terms and most of the transitions observed in the UV and EUV is being carried out.

\section{Discussion}

The present status of the second phase of the IP regarding collisional and radiative data for the low ionization stages of Iron is reported. Photoionization cross sections and transition probabilities for Fe II are already available and the corresponding data for $\mathrm{Fe} \mathrm{I}$ and Fe III-V is expected to become available in the near future. Collision strengths for Fe II and Fe III have been calculated and may be used for the analysis of observed spectra. Collisional data for Fe IV-VI is currently being carried out.

The third phase of the IP that deals with Iron peak elements and ions other than Iron is currently in progress and, for instance, the first results for $\mathrm{Ni}$ II have recently been obtained (Bautista \& Pradhan 1995b). Extensive ab initio calculations for the Iron peak elements and ions is difficult and requires hundreds to thousands of CPU hrs on modern supercomputers. For this reason we are investigating the capabilities of new technologies such as massive parallel processors (MPP). The first of these calculations on the MPP CRAY T3D are reported by Bautista \& Pradhan (1995b).

This work is partially supported by the U.S. National Science Foundation (PHY9115057), NASA LTSA program (NAGW-3315), and NASA ADP program (NAS 532643). The computational work is carried out on the CRAY Y-MP8/64 and the MPP CRAY T3D at the Ohio Supercomputer Center in Columbus, Ohio.

\section{REFERENCES}

Bautista, M. A. \& Pradhan, A. K. 1995a, Photoionization of neutral Iron, J. Phys. B Lett., in press

Bautista, M. A. \& Pradhan, A. K. 1995b, Atomic Data from the Iron Project XIV, Electron excitation rates and emissivity ratios for forbidden transition in Ni II and Fe II, A\&A, submitted

Berrington, K. A., Burke, P. G., Hibeert, A., Mohan, M., \& Baluja, K. L. Electron impact excitation of $\mathrm{Fe}^{+}$using the R-matrix method incorporating fine-structure effects, J. Phys. B: At. Opt. Phys., 21, 339

Berrington, K. A., Zeippen, C. J., Le Dourneuf, M., Eissner, W., \& Burke, P. G. Electron impact excitation of $\mathrm{Fe}^{2+}$ : I. A 17-level fine-structure calculation, J. Phys. B: At. Opt. Phys., 24, 3467

Bell, R. A., Paltoglou, G., \& Tripicco, M. J. 1994, The calibration of synthetic colours, MNRAS, 268, 771

Cunto, W., Mendoza, C., Ochsenbein, F., \& Zeippen, C. J. TOPbase at the CDS, A\&A, 275, L5 
Hummer. D. G., Berrington, K. A., Eissner, W., Pradhan, A. K., Saraph, H. E., \& TUlly, J. A. 1993, Atomic data from the IRON Project, A\&A, 279, 298

Keenan, F. P., HibBert, A., Burke, P. G., \& Berrington, K. A. 1988, Fine-structure populations for the ${ }^{6} \mathrm{D}$ ground state of Fe II, ApJ, 332, 539

KuRUCz, R. L. 1981, Semiempirical calculation of gf values: Fe II, Smithsonian Astrophysical Observatory Special Report 390, Cambridge

KuRUCZ, R. L. 1991, private communication

NAHAR, S. N. 1995, Atomic Data from the Iron Project VII. Radiative dipole transitions probabilities for Fe II, A\&A, 293, 967

Nahar, S. N. \& Pradhan, A. K. 1994, Atomic data for opacity calculations: XX. Photoionization cross sections and oscillator strengths for Fe II, J. Phys. B: At. Opt. Phys., 27, 429

NAHAR, S. N. \& PRADHAN, A. K. 1995, Unified electron-ion recombination rate coefficients of Silicon and Sulfur ions, ApJ, in press

Pradhan, A. K. 1995, these proceedings

ReILMAN, R. F. \& Manson, S. T. 1979, Photoabsorption cross section for positive atomic ions with $\mathrm{Z} \leq 30$, AJSS, 40, 815

SAWEY, P. M. J. \& BerRington, K. A. 1992, Atomic data for opacity calculations: XV. Fe I-IV, J. Phys. B: At. Opt. Phys., 25, 1451

Seaton, M. J., Zeippen, C. J., Tully, A. K., Pradhan, A. K., Mendoza, C., Hibbert, A., \& BerRington, K. A. 1992, The Opacity project-Computation of atomic data, Rev. Mexicana Astron. Astrof., 25, 19

VENNES, S. 1995, these proceedings

Verner, D. A., Yakovlev, D. G., Band, I. M., \& Trzhaskovskaya, M. B. 1993, At. Data Nucl. Data Tables, 55, 233

Zhang, H. L. \& Pradhan, A. K. 1995a, Atomic Data from the Iron Project VI. Collision strengths and rate coefficients for Fe II, A\&A, 293, 953

Zhang, H. L. \& Pradhan, A. K. 1995b, Relativistic and electron correlation effects in electron impact excitation of $\mathrm{Fe}^{2+}$, J. Phys. B: At. Opt. Phys., submitted 